\title{
EL ROL DEL CONCILIADOR EN EL JUZGADO ESPECIAL CIVIL ESPECIAL DEL CUARTO JUZGADO ESPECIAL CIVIL DE MACAPÁ- AP, AMAZÔNIA, BRASIL
}

\section{ARTICLE ORIGINAL}

NETO, Dalk Dias Salomão', SOUSA, Nicole Moreira Faria², DENDASCK, Carla Viana $^{3}$, FECURY, Amanda Alves ${ }^{4}$, OLIVEIRA, Euzébio de ${ }^{5}$, DIAS, Claudio Alberto 6

NETO, Dalk Dias Salomão. Et al. El rol del conciliador en el Juzgado Especial Civil Especial del Cuarto Juzgado Especial Civil de Macapá-AP, Amazônia, Brasil. Revista Científica Multidisciplinar Núcleo do Conhecimento. Año 06, Ed. 05, Vol. 11, pp. 80-92. Mayo de 2021. ISSN: 2448-0959, Enlace de acceso: https://www.nucleodoconhecimento.com.br/ley/juzgado-especial, DOI: 10.32749/nucleodoconhecimento.com.br/ley/juzgado-especial

\section{RESUMEN}

Debido a numerosos problemas sociales, la sociedad comenzó a pedir más justicia. Era urgente un nuevo paradigma, con la creación de cortes especiales, basados en la conciliación, como medio alternativo de resolución de conflictos como solución parcial. Los tribunales especiales han evolucionado mucho desde su creación, a través de la ley 9.099/95, que resultó extremadamente importante para la reorganización de la justicia, frente a las grandes demandas de los juicios. El objetivo de este trabajo era analizar el papel del conciliador en el tribunal civil virtual especial en el 4avara de la

\footnotetext{
${ }^{1}$ Abogado, Licenciado en Derecho (CEAP - Centro de Educación Superior de Amapá), Especialista en Derecho Laboral y Proceso Laboral por la institución educativa Damásio.

${ }^{2}$ Abogado, Licenciado en Derecho (CEAP - Centro de Educación Superior de Amapá), especialista en Derecho Procesal Civil por la institución Damásio Educacional.

${ }^{3}$ Teóloga, Doctora en Psicoanálisis Clínica. Trabaja desde hace 15 años con Metodología Científica (Método de Investigación) en Orientación de Producción Científica para Estudiantes de Maestría y Doctorado. Especialista en Investigación de Mercados e Investigación en Salud, Estudiante de Doctorado en Comunicación y Semiótica (PUC SP).

${ }^{4}$ Biomédico, Doctor en Enfermedades Tropicales, Profesor e investigador del Curso Médico del Campus Macapá, Universidad Federal de Amapá (UNIFAP), Pro-Rector de Investigación y Estudios de Posgrado (PROPESPG) de la Universidad Federal de Amapá (UNIFAP).

${ }^{5}$ Biólogo, Doctor en Enfermedades Tropicales, Profesor e investigador del Curso de Educación Física de la Universidad Federal de Pará (UFPA).

${ }^{6}$ Biólogo, Doctor en Teoría e Investigación del Comportamiento, Profesor e investigador del Curso de Grado de Química del Instituto de Educación Básica, Técnica y Tecnológica de Amapá (IFAP) y del Programa de Posgrado en Educación Profesional y Tecnológica (PROFEPT IFAP).
}

RC: 85943

Enlace de acceso: https://www.nucleodoconhecimento.com.br/ley/juzgado-especial 
corte civil especial de Macapá AP, Amazônia, Brasil. Se concluye que hubo una evolución histórica de los jueces especiales, desde su aplicación a través de la Ley 9.099/95 hasta la construcción de sus principios como la rapidez procesal, la informalidad y, todo apuntando a la realización de un proceso más rápido y eficiente. PROJUD y TUCUJURIS computarizando las canchas especiales parecen ser importantes en la modernización del proceso, haciéndolos más simples, rápidos y accesibles para todos y siguiendo así las tendencias tecnológicas del mundo. Los tribunales especiales en su conjunto deben tratar de invertir cada vez más en conciliadores y acciones encaminadas a la conciliación, como las semanas de conciliación estatales y nacionales, porque por lo tanto, habrá una gran posibilidad de que los casos en el poder judicial ganen mayor fluidez.

Palabras clave: Tribunal Civil Especial, Conciliación, Conciliador.

\section{INTRODUCCIÓN}

Debido a numerosos problemas sociales, la sociedad comenzó a pedir más justicia, teniendo problemas, como el gran número de demandas procesales, el retraso en los procesos judiciales y la burocracia (Sena e Richa, 2011; Guimarães, 2013).

Sumando estas situaciones con la dificultad de acceso de personas con hiposuficiencia económica en el poder judicial, era urgente la necesidad de un nuevo paradigma, teniendo como solución parcial la creación de tribunales especiales, basados en la conciliación, como medio alternativo de resolución de conflictos (Sérpias, 2015).

Los Tribunales Especiales del Brasil, anteriormente llamados Tribunal de Reclamos Menores, modelaron una estructura estadounidense conocida como Small Claims Courts o corte pequeño. Creado en 1934 en Nueva York, con el objetivo de disfrutar de acciones civiles de pequeño valor. La característica principal de este modelo de corte es el pragmatismo propio de la cultura estadounidense, es decir, el conflicto visto se eleva a una perspectiva más rápida, sencilla y fácil. No era necesaria la figura del 
abogado, que fue solicitado en función de la complejidad de la situación (Oporto, 2008).

Este modelo de corte apareció en Brasil, primero en Río Grande do Sul, en 1982, antes del funcionamiento de los Consejos de Conciliación y Arbitraje, creados con el objetivo de resolver, extrajudicialmente, conflictos de pequeñas causas, demostrando un gran interés por la construcción de una Justicia (Silva, 2013).

Después de ganar protagonismo en el escenario nacional, en 1984, el Poder Judicial tuvo como hito la Ley № 7.244, que prevé la creación y funcionamiento de tribunales de pequeñas causas, consolidándose y legitimando el éxito de la experiencia obtenida con los Consejos de Conciliación y Arbitraje, teniendo como deber, proporcionar una justicia igualitaria (Silva, 2013).

A partir de ese momento, varios procedimientos de la Corte Especial están empezando a surgir en todo el país, regulados en la oficina estatal por la Ley Ordinaria, que cubren sólo causas civiles de pequeño valor económico, estipulados por primera vez en hasta veinte (20) salarios mínimos. Así, también permitió a los más desprovistos de la oportunidad y la garantía jurídica buscar el poder judicial para obtener una resolución pacífica de sus conflictos (Silva, 2013).

La Constitución Federal de 1988 demostró la necesidad real de crear jueces especiales, y su implementación en todo el país, siendo una medida obligatoria, ya que anteriormente era opcional para los estados. El CF aseguró a través de su artículo 98, punto I, la autorización para implementar los Tribunales Civiles Especiales para conciliar, enjuiciar, juzgar y ejecutar los casos civiles de menor complejidad, así como los tribunales penales especiales para conciliar, juzgar y ejecutar delitos de menor potencial ofensivo, mediante procedimientos orales y sumaríssimo (que não há atrasos, nem formalidades) (Sérpias, 2015).

Desde la creación de la Ley Complementaria № 123/2006, con el objetivo de satisfacer los deseos de los micro y pequeños empresarios sobre la constitución y regulación de me y PPE, con el fin de expandir los negocios y desarrollar su crecimiento en la economía brasileña. Así, el ME y el PPE ganaron espacio en el escenario nacional, y RC: 85943

Enlace de acceso: https://www.nucleodoconhecimento.com.br/ley/juzgado-especial 
a partir de esta ley general, se creó la disposición jurídica sobre la posibilidad de que estas especies de empresas, a nivel estatal, tuvieran derecho a entrar en el Tribunal Civil Especial, apareciendo en el polo activo de la acción (Salomão, 2001). Dada la posibilidad de acceder a un tribunal especial, estas empresas pueden ingresar sus acciones sin la presencia de un abogado (con un tope para el valor de la acción de hasta 20 salarios mínimos), reduciendo sus gastos. Es importante señalar que los casos en los Tribunales Especiales se manejan más rápidamente que en el tribunal común, permitiendo más rápidamente la recuperación de capital, que hasta entonces se perdía por impago de deudores (Tolentino, 2011).

En 2001, poco después de la creación de la Ley 9.099/95 (Ley de Tribunales Especiales), se creó la ampliación del tribunal civil para atender a las microempresas en Amapá, por lo que no podían exigir en el Tribunal Especial Central, que era el único en funcionamiento en cualquier estado en ese momento, teniendo como coordinadora a la magistrada Sueli Pini, actual presidenta del Tribunal de Justicia de Amapá (Serra, 2015).

Poco después de la creación del Estatuto Nacional del ME y del EPP, se creó la Ley Estatal Complementaria № 044/2007, donde se pone de acuerdo con que los beneficios de la ley № 123/06 fueron aceptados por el Gobierno de Amapá, el Tribunal Especial de Microempresas y Pequeñas Empresas fue finalmente creado en marzo de 2012 (Serra, 2015).

El tribunal de microempresas, en Amapá, tiene un papel relevante a través del trabajo desarrollado y el número de reconciliaciones fructíferas. La mayoría de los casos, ya archivados, se deben a la aprobación de acuerdos, tanto judicial como extrajudicialmente. Contar principalmente con la ayuda de sus colaboradores en prácticas, que en la mayoría de los casos son los conciliadores en las audiencias. Es importante señalar que el juzgado $4^{\circ}$ del Tribunal Civil Especial de Macapá es el que tiene más prácticas de nivel superior, en vista de sus alianzas firmadas como TJAP, Fecomércio, FEMICRO y SEBRAE (Serra, 2015). 
Por lo tanto, los tribunales especiales han evolucionado mucho desde su creación, a través de la ley 9.099/95, que resultó extremadamente importante para la reorganización de la justicia, frente a las grandes demandas de las demandas. Así como la valorización del ME y el PPE, ganando espacio ante los tribunales especiales, para luchar de una manera más digna por sus derechos, siendo el juzgado número 4 de la corte civil especial de Macapá (antiguo tribunal de microempresas y pequeña empresa de Macapá), pionero en Brasil.

\section{GOL}

Analizar el papel del conciliador en la corte civil virtual especial en el 4avara de la corte civil especial de Macapá AP, Amazon, Brasil.

\section{MÉTODO}

Investigación cuantitativa y cualitativa, utilizando datos del Juzgado $4^{\circ}$ de la Corte Civil Especial de la ciudad de Macapá, Amapá, Amazônia, Brasil, sobre conciliación en procesos judiciales.

\section{RESULTADOS Y DISCUSIÓN}

Los Tribunales Civiles Especiales son órganos que integran la Justicia Ordinaria, y fueron creados con el propósito de conciliación, proceso, juicio y ejecución, en las causas de su jurisdicción, disciplinados por la Ley Federal № 9,099 de 26 de septiembre de 1995. Las bases de este procedimiento se guían por los criterios de oralidad, sencillez, informalidad, economía procesal y rapidez, con el objetivo, siempre que sea posible de conciliación, la reparación del daño sufrido por la víctima y la aplicación de una pena no privativa de libertad (Neto y Júnior, 2011).

La verdadera intención de los tribunales civiles especiales es la resolución de los problemas de acceso a la justicia, básicamente relacionados con los costos y el retraso de los casos, y también hay numerosos obstáculos que los ciudadanos, que piden justicia, encuentran (Guimarães, 2013). 
Los Jueces Civiles Especiales están cada vez más presentes en la vida de toda la población, ya que su propia existencia está justificada. Se presenta por su forma moderna de actuar, por la calidad del servicio y por los intereses de sus dirigentes que los hace cada vez más ágiles y accesibles (Bacellar, 2003).

El Tribunal Civil Especial es competente para la conciliación, los procedimientos y la sentencia de casos civiles de menor complejidad, son ejemplos: casos cuyo valor no supera cuarenta veces el salario mínimo; los enumerados en el art. 275, punto II, del Código de Procedimiento Civil; la acción de desalojo para su propio uso; acciones de posesión en bienes inmuebles de valor no superiores a cuarenta veces el salario mínimo; valores ejecutivos extrajudiciales (pagarés, cheques, contratos), por un importe de hasta cuarenta veces el salario mínimo; recaudación y indemnización por daños y perjuicios (por ejemplo, daños resultantes de accidentes de tráfico) (Barouche, 2010).

\section{PRINCIPIOS DE JUECES CIVILES ESPECIALES}

La creación de jueces especiales es un hito importante para el mundo jurídico en todos sus ámbitos, por lo que es necesario destacar los principios que guían este orden, tanto en la búsqueda de su significado, como en el alcance y aplicabilidad de la norma legal (Porto, 2008).

Según Sérpias (2015) "el objetivo de la creación de un tribunal civil especial es resolver rápida y económicamente los pequeños problemas del ciudadano en el día a día". Por lo tanto, la ley se creó sobre la base de principios compatibles con la facilitación del acceso a la justicia de quienes la solicitan.

\section{PRINCIPIO DE ORALIDAD}

Debido al molde moderno de jueces especiales, el principio de oralidad destaca por su fácil aplicabilidad. Este principio se incluye entre los generales del procedimiento civil, con el objetivo de prevalecer los actos procesales a través de la comunicación oral, aunque estos actos pueden reducirse a escrito (Silva, 2012). 
En los cortesanos especiales este principio está mucho más presente, y sólo lo esencial se reduce a término. El resto puede estar grabado en cinta magnética o equivalente, de conformidad con el Artículo 13, §3, de la Ley N 9,099/95 (Brasil, 1995). Esto demuestra que la oralidad no ha sustituido a la escritura, que van de la mano, ya que es esencial convertir el proceso y la conversión de sus actos procesales en término.

La aplicación de este principio también tiene por objeto que los actos procesales se realicen en una sola etapa o en momentos aproximados. Por lo tanto, este principio se refiere directamente a la velocidad procesal, ya que no debería haber una brecha horaria muy grande entre las prácticas procesales (Silva, 2012). Por lo tanto, este principio busca la simplicidad procedimental, como medio para facilitar el progreso del proceso, culminando en su rapidez.

\section{PRINCIPIO DE INFORMALIDAD Y SENCILLEZ}

El propio nombre de estos principios ya demuestra su intención, pretende facilitar el proceso y el acceso al poder judicial, no manteniendo la propia forma, sino más bien una mayor flexibilización de los actos procesales, validándolos siempre que alcancen su propósito, según (art. 13 de la Ley 9.099/95): "Arte. 13 - Los actos procesales serán válidos siempre que cumplan los fines para los que se realicen, cumpliendo los criterios indicados en el art. 2 de la presente Ley" (Brasil, 1995)

El principio de simplicidad no es más que un desdoblamiento del principio de informalidad, que a su vez es el intento de hacer que los procedimientos judiciales sean más informales, sin esa exacerbación de los formalismos y las burocracias. Según Bobbio, el celo por la burocracia procesal en lugar de garantizar la plena eficacia de la disposición judicial terminó resultando en el retraso del procedimiento (Porto, 2008).

Un ejemplo práctico de estos principios es la forma en que se hace la citación postal de las personas jurídicas del derecho privado, siendo válida con la simple entrega de correspondencia a cualquier empleado a cargo de la recepción (art. 18, punto II, de la 
Ley No. 9.099/95), a diferencia del Código de Procedimiento Civil que condiciona la entrega a determinadas personas, por ejemplo, a aquellas que tienen competencias de gestión o administración; (art. 18, § 2 de la Ley 9.099/95) (Brasil, 1995): "Arte. 18 - La cita se efectuará: [...] II - en el caso de una persona jurídica o empresa individual, mediante entrega al responsable de la recepción, que se identificará necesariamente".

Por lo tanto, estos dos principios con el foco principal de llevar a la población más simple al foro, ya que la parte más desfavorecida de las personas y laicos en los asuntos legales se siente tímida ante abogados, jueces, formalismos y personas con vocabularios más descabellados.

\section{PRINCIPIO DE ECONOMÍA PROCESAL}

Otro principio es el de la economía procesal, que a su vez es una guía no sólo del tribunal especial, sino de la teoría general del proceso en su conjunto, ya que a través de ella tiene como objetivo principal lograr tantos resultados con el menor gasto económico posible (Sérpias, 2015).

Por lo tanto, este principio tiene por objeto el mayor número de actos procesales que se realizan en el menor tiempo posible y de la manera menos costosa posible.

\section{PRINCIPIO DE VELOCIDAD PROCESAL}

En cuanto al principio de rapidez, se trata de garantizar la eficacia de la justicia, con el objetivo de hacer los efectos de una sentencia, por ejemplo, de forma práctica y rápida y no de una manera lenta como ocurre en muchos casos, especialmente fuera de los tribunales especiales (Sérpias, 2015)

Según Sérpias (2015) "El retraso en la disposición judicial se ha convertido en una regla cuando debería ser una excepción. El retraso del final del proceso fue institucionalizado"." Por lo tanto, los otros principios no son más que una conversión al principio de velocidad, con el objetivo de hacer el proceso más rápido. 
Por lo tanto, sin duda, la mayor expectativa generada por la Ley 9.099/95 es la promesa de velocidad sin violar el principio de seguridad de los drenajes legales. Con la intención de hacer el proceso más ágil y rápido, y sólo así lograr su principal propósito, una respuesta eficiente de la provisión judicial a la sociedad.

\section{INFORMATIZACIÓN DE JUECES CIVILES ESPECIALES- PROJURD}

El mundo ha experimentado un gran avance tecnológico en los últimos años, aliado con la falta de espacio físico para proteger numerosos procesos, especialmente después de la constitución de 1988 y la internacionalización de la economía con la creación de los factores reales y que aprovecharon el número de demandas en el poder judicial. Con esto, el poder judicial brasileño creó la sociedad PROJUDI (proceso judicial digital) (Silva, 2012).

El objetivo principal de la creación de PROJUDI tiene como objetivo agilizar la justicia, reducir costes, aumentar la capacidad de tramitación de las acciones, facilitar el trabajo de los abogados y mejorar la calidad del servicio a las partes. Así, puede acabar con esos estantes llenos de papeles, ya que en décadas anteriores los procesos se hicieron casi artesanales, permitiendo una reducción del tiempo de espera para la apreciación de las solicitudes, buscando siempre resolver conflictos más rápidamente sin causar daño a las partes.

La verdadera revolución comenzó a través de la Ley 11.419/2006, con la intención de disciplinar el proceso electrónico, con profundos cambios en el Código de Procedimiento Civil, y con miras a racionalizar, impulsar, acortar el camino creado por la burocracia y la distancia siempre común en los procesos. Podemos ver que dos campos han evolucionado significativamente con la informatización, agilidad y velocidad procesal, porque todos los procesos se transmiten a través de Internet sin tener que salir de la oficina (Carvalho, 2006).

En el tema de la informatización, el estado de Amapá fue más allá, en 2005, creó el sistema TUCUJURIS, un dispositivo tecnológico que busca gestionar y desarrollar las actividades del poder judicial electrónicamente. Informatizar los procedimientos 
judiciales y realizar en las Secretarías Judiciales, así como el almacenamiento de los contenidos de los diversos actos procesales, con el objetivo de una mayor agilidad de los servicios, sustituyendo al entonces sistema actual (Tjap, 2016).

El sistema TUCUJURIS ha ido mejorando a lo largo de los años, y sus procedimientos han mejorado cada vez más, con adiciones de nuevas características que lo convierten en un software más integrado dentro del ámbito de la justicia, diversificando su operacionalización, con el objetivo de dar mayor eficiencia a la ejecución de actos jurisdiccionales por parte de los Servidores, Magistrados, Abogados Defensores y Fiscales, así como una respuesta más rápida a otros miembros de la sociedad que utilizan este servicio (Tjap , 2016).

Por lo tanto, la informatización es indispensable para el mantenimiento de una justicia rápida y eficaz, porque hace que el poder judicial sea moderno, siguiendo las tendencias tecnológicas de hoy.

\section{CONCILIACIÓN EN LOS JUECES ESPECIALES CIVEIS}

El instituto de conciliación es conocido por la gran mayoría de los brasileños. Sin embargo, su aplicación en el ámbito del derecho, vinculada a cuestiones aplicadas a la justicia conciliadora, sigue siendo nueva para muchos (Guimarães, 2013).

Aunque la conciliación ha estado presente en el sistema legal brasileño desde la época del imperio, fue en las últimas décadas que este instituto ganó notoriedad. Desde la emancipación política de Brasil en 1822, la constitución del imperio ha alentado abiertamente la resolución de conflictos a través de medios no judiciales, y la conciliación es una condición preventiva e indispensable para el procesamiento de cualquier causa, con la figura de la justicia de la paz como principal medio para resolver el litigio (Silva, 2013).

En 1982, se publicó la ley de los tribunales especiales de pequeñas causas, con el objetivo de desbureaucratizar el poder judicial a nivel nacional. Creado en 1984 definitivamente por la Ley 7.244/84 (Sérpias, 2015). 
Con la promulgación de esta ley, la conciliación vuelve a tener protagonismo en la resolución de conflictos, incluida la inserción de la figura del conciliador, que se estudiará más adelante (Guimarães, 2013).

La conciliación es un método utilizado en conflictos menos complejos, en el que el tercer facilitador puede adoptar una posición más activa pero neutral con respecto al conflicto, no perdiendo imparcialidad. Se trata de un breve proceso consensuado que busca una resolución efectiva de los conflictos, dentro de los posibles límites, de la relación social entre las partes implicadas (Sérpias, 2015).

En resumen, "la conciliación es un instituto del proceso, porque es uno de sus momentos que produce efectos procesales, privilegia el principio curioso y el papel del juez interviniente en la formación de la voluntad de las partes" (Nassif, 2005).

La conciliación es un proceso autocomplaciente en el que un tercero imparcial (el conciliador) asiste a las partes en el conflicto para encontrar una solución satisfactoria para todas las partes (Silva, 2013).

La conciliación en el juzgado $4^{\circ}$ de lo civil, está bastante desarrollada y practicada, siendo un tribunal de este tipo conocido a nivel nacional por el trabajo desarrollado y el número de reconciliaciones positivas. La mayoría de los casos ya archivados son fruto de la aprobación de acuerdos, tanto judicial como extrajudicialmente (Serra, 2015).

Podemos concluir entonces que el instituto de conciliación es, sin duda, el medio alternativo de resolución de conflictos más utilizado y conocido en brasil, lo que permite a la justicia resolver rápidamente sus demandas, así como hacer realidad la posibilidad de una resolución pacífica de lides.

\section{PASOS DE CONCILIACIÓN}

La conciliación, por informal que sea, tiene algunas medidas que deben ser observadas por el conciliador en la construcción de un acuerdo, pasos que no son 
reglas. Sin embargo, el cumplimiento de estos pasos ayuda en la velocidad y eficacia del proceso (Sérpias, 2015).

Por lo tanto, la conciliación tiene directrices que deben cumplirse, y no deben suceder por la simple voluntad de las partes o del conciliador. A continuación mencionaremos las principales etapas de conciliación.

\section{LO CONCILIADOR Y SUS CARACTERÍSTICAS EN EL TRIBUNAL CIVIL VIRTUAL}

La pregunta interesante en este momento es la figura del conciliador en los procedimientos de la Ley 9.099/95. Los conciliadores serán preferiblemente reclutados de estudiantes legales. Así que si la ley decía preferiblemente, no lo hace una obligación. Por lo tanto, los estudiosos del derecho también pueden figurar como conciliadores. Según la Ley 9.099/95: "Arte. 7. Los conciliadores y jueces laicos son auxiliares de justicia, reclutados, el primero, preferiblemente, entre los estudiantes de derecho, y el segundo, entre abogados con más de cinco años de experiencia" (Brasil, 1995).

En la práctica, la gran mayoría de los conciliadores del Tribunal Civil Especial son estudiantes de derecho, extraídos de universidades generalmente después de entrar en el quinto semestre. Ser una asociación, donde se cambia el trabajo por experiencia, no ser diferente en el juzgado 4 de la Corte Civil Especial (Serra, 2015).

El conciliador es uno de los temas de conciliación, y debe actuar de manera neutral e imparcial, creando un momento y una situación cuyas partes pueden sentirse cómodas para negociar. Debe utilizar su psicología para hacer fructífera la conciliación, a través de las voluntades de las partes en lados opuestos, es decir, el conciliador no es más que un facilitador (Porto, 2008).

Aun cargando toda la carga emocional, durante la audiencia de conciliación el conciliador debe ser lo más imparcial posible y neutralmente, debe garantizar la suavidad y legitimidad del proceso (Silva, 2013). La imparcialidad del conciliador es fundamental para la conciliación, esto significa que no privilegiará a ninguna de las RC: 85943

Enlace de acceso: https://www.nucleodoconhecimento.com.br/ley/juzgado-especial 
partes, y mucho menos emitirá juicio de valor relacionado con el conflicto. Por lo tanto, debe transmitir confianza a las partes. Sólo así es posible construir un acuerdo justo para todos (Amaral, 2011).

En relación con el juzgado $4^{\circ}$ de la Corte Civil Especial, siendo muy famoso por el número de reconciliaciones fructíferas, obtenidas en audiencias diarias o eventos celebrados esporádicamente como: semana de conciliación estatal o nacional y "el sábado también es día conciliador", destaca por la eficiencia de sus conciliadores, y la mayoría son aprendices de mayor nivel de derecho, dadas sus asociaciones firmadas entre el propio tribunal y el TJAP, Fecomércio, FEMICRO y SEBRAE (Serra, 2015).

Por lo tanto, la figura del conciliador frente a la conciliación es fundamental, porque él es el que organiza y lleva a cabo el trabajo. Debe actuar de conformidad con la ley y las directrices a observar.

\section{LA EFICIENCIA DE LA CONCILIACIÓN}

No sólo la falta de eficacia y acelerar los principales problemas de la justicia, otras situaciones ayudan a retrasar y detener el poder judicial. Por lo tanto, a través de la Constitución Federal de 19888 y regulada por la ley 9.099/95, los tribunales civiles especiales surgieron como una forma de ampliar el acceso a la justicia y se caracterizó como una "válvula de escape" a la justicia común, cambiando los procedimientos tradicionales que eran ineficaces, especialmente para la resolución de pequeños conflictos (Guimarães, 2013).

La creación de tribunales especiales y el uso de la conciliación tiene una eficacia mucho más allá de lo esperado, porque además de desahogue el poder judicial, puso en el ámbito de la justicia a los ciudadanos que hasta entonces no utilizaban este medio para resolver sus conflictos, difundiendo así la ley y la cultura de la paz (Guimarães, 2013).

Así, a lo largo de los años la conciliación ha demostrado ser efectiva, ya que desenviva al poder judicial, mediante la reducción de costes, plazos y resolución de causas de RC: 85943

Enlace de acceso: https://www.nucleodoconhecimento.com.br/lev/juzgado-especial 
menor complejidad, creando nuevos parámetros para la resolución de conflictos en el conjunto de la justicia, teniendo en cuenta la rapidez (Guimarães, 2013).

Por lo tanto, la mayor velocidad de los jueces especiales se basa en la idea de velocidad y eficacia, y hay dos maneras de subdividir la eficiencia, cualitativa y cuantitativa. La cuantitativa no se refiere a la calidad procesal, teniendo en cuenta únicamente la rapidez de los procedimientos y la reducción de costes, mientras que cualitativa, en sentido contrario, se refiere al contenido de las decisiones, teniendo en cuenta su calidad en cuanto al cumplimiento y adopción del proceso democrático, con todas las normas inherentes a él (Neto, 2015).

En este sentido, podemos decir que aunque los tribunales especiales y la conciliación son eficaces, dar celeridad a sus procedimientos no significa que los problemas del poder judicial hayan sido resueltos, mucho menos podemos decir que la justicia en su conjunto es rápida (Barouche, 2010).

En relación con Macapá, los jueces especiales son muy eficaces, dado el incentivo a la conciliación (Serra, 2015).

Por ejemplo, el juzgado número 4 de la corte especial de Macapá, antiguo tribunal de microempresas y pequeñas empresas, tiene un trabajo muy concreto y eficaz mediante la conciliación, siendo reconocido a nivel nacional por su trabajo que resulta anualmente en conciliaciones fructíferas (Serra, 2015).

Durante el año, se llevan a cabo numerosas acciones de conciliación, como semanas de conciliación estatales y nacionales, por ejemplo. En ellos, el juzgado número 4 de la Corte Civil Especial de Macapá es siempre como los que hacen las reconciliaciones más fructíferas (Serra, 2015).

La parte física del tribunal sin duda limita en gran medida el número de conciliaciones, pero aún así, se celebran diariamente entre 12 y 25 audiencias de conciliación, un número que es un número mayor en comparación con los otros tribunales especiales del tribunal central de Macapá (Serra, 2015). 
Figura 1 - Muestra el número de casos relativos a conciliación, instrucción y juicio

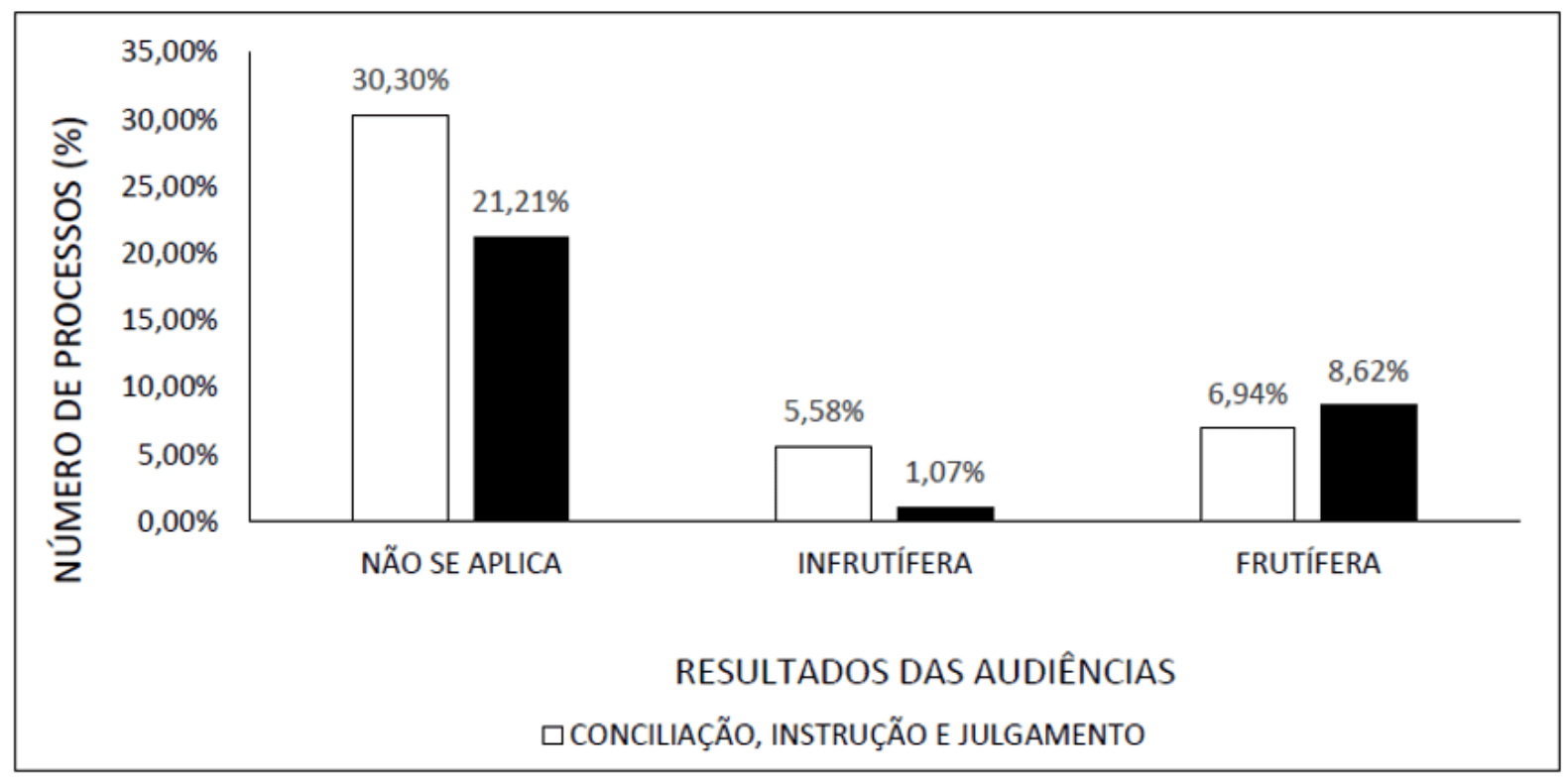

(Serra, 2015).

La Figura 1 muestra la eficacia de las audiencias de conciliación celebradas en el juzgado 4 del Tribunal Civil Especial de Macapá. Durante las audiencias inaugurales de conciliación, el porcentaje de acuerdos fructíferos es mayor; y el acuerdo fallido es menor (dada la presencia del conciliador), en relación con la etapa de instrucción y juicio, ante el juez.

Por lo tanto, podemos concluir que el objetivo de la creación de tribunales especiales se logró en el juzgado 4ํํㄹ del tribunal civil especial de Macapá, porque, a través de la conciliación, el número de demandas resueltas a través de acuerdos es significativo en relación con las demás fases procesales. Mostrando así la eficacia del conciliador como motor de las audiencias de conciliación.

\section{CONCLUSIONE}

En vista del aumento del número de casos, el poder judicial parece haberse congestionado e ineficiente, y es necesario buscar nuevos medios para la resolución de conflictos. La respuesta del poder judicial fue la creación de tribunales especiales mediante la conciliación. 
Ha habido una evolución histórica de los jueces especiales, desde su aplicación a través de la Ley 9.099/95 hasta la construcción de sus principios como la rapidez procesal, la informalidad y, todo apuntando a la realización de un proceso más rápido y eficiente.

PROJUD y TUCUJURIS mediante la informatización de canchas especiales parecen ser importantes para modernizar el proceso, haciéndolos más simples, más rápidos y accesibles para todos y siguiendo así las tendencias tecnológicas globales

El instituto de conciliación resulta ser el principal medio alternativo de resolución de conflictos, una forma de autocompontura, por la cual las partes crean la solución a los problemas, desbancar al poder judicial a través de la rapidez.

La conciliación a través de la velocidad, los bajos costos y la eficiencia parecen hacer que el poder judicial sea más atractivo para una parte de la sociedad de bajos ingresos y para los laicos en el tema, que creen en la posibilidad de resolver sus problemas detrás de la justicia, generando así efectos positivos en la pacificación social.

La evolución del procedimiento especial, basado en la conciliación, no sería efectiva sin la presencia del conciliador. Es él quien dirige a las partes en la redacción de un acuerdo, llevando a cabo imparcialmente, guiando el procedimiento sobre numerosos principios y directrices. Por lo tanto, debería haber una mayor preocupación con la formación de estos agentes, ya que son los que están a la vanguardia de la mayoría de las demandas del poder judicial, tratados directa y diariamente con la población y sus problemas, en situaciones estresantes y, a veces, difíciles de conciliar.

Los tribunales especiales en su conjunto deben tratar de invertir cada vez más en conciliadores y acciones encaminadas a la conciliación, como las semanas de conciliación estatales y nacionales, porque por lo tanto, habrá una gran posibilidad de que los casos en el poder judicial ganen mayor fluidez. 


\section{REFERENCIAS}

AMARAL, R. L. S. D. A Concilição no Processo Judicial, . 2011. 64 (Graduação). Faculdade de Ciências Jurídicas da Universidade Tuiuti do Paraná, Curitiba PR.

BACELLAR, R. P. Juizados Especiais - a Nova Mediação Paraprocessual. São Paulo: Revista dos Tribunais, 2003. 286.

BAROUCHE, T. D. O. Os Juizados Especiais Cíveis e a Problemática da Celeridade Processual. Revista de Direito dos Monitores da UFF, n. 7, p. 1-24, 2010.

BRASIL. LEI № 9.099, DE 26 DE SETEMBRO DE 1995. JURÍDICOS, S. P. A. Brasília DF: Presidência da República - Casa Civil 1995.

CARVALHO, J. P. C. D. A Era Virtual do Processo Judicial: A Experiência dos Juizados Especiais Virtuais e o Projeto de Lei de Informatização do Processo. Revista Direito e Liberdade, v. 3, n. 2, p. $453-484,2006$.

GUIMARÃES, A. A Crise do Judiciário e a Eficácia da Conciliação nos Juizados Especiais Cíveis Estaduias de São Luis - MA. Revista Thesis Juris, v. 2, n. 1, p. 142$161,2013$.

NASSIF, E. Conciliação judicial e indisponibilidade de direitos: paradoxos da "justiça menor" no processo civil e trabalhista. São Paulo SP: Editora LTR, 2005.

NETO, A. G. A Audiência de Conciliação no Novo Código de Processo Civil. Revista Eletrônica de Direito Processual - REDP. 16, n. 16, p. 29-57, 2015.

NETO, F. D. C. T.; JÚNIOR, J. D. F. Juizados Especiais Estaduais Cíveis e Criminais. Comentários à Lei no 9.099/95. $7^{a}$ ed. São Paulo: Revista dos Tribunais, 2011. 960p.

PORTO, A. P. G. Juizados Especiais Cíveis - O Papel do Conciliador 2008. 59p. (Especialização). Universidade Estadual Vale do Acaraú, Fortaleza CE. 
SALOMÃO, L. F. As Microempresas nos Juizados Especiais. Revista da EMERJ, v. 4, n. 4, p. 62-73, 2001.

SENA, A. G.; RICHA, M. D. A. A conciliação judicial trabalhista em uma política pública de tratamento adequado e efetivo de conflito de interesses. In: PELUSO, A. C. e RICHA, M. D. A. (Ed.). Conciliação e mediação: estruturação da política judiciária nacional. Rio de janeiro RJ: Forense, 2011. p.115-139.

SÉRPIAS, L. Juizado Especial, Cível, Criminal, Federal e da Fazenda Pública. São Paulo: TradeBook, 2015. 1615p.

SERRA, A. B. C. S. Juizado Especial da Microempresa e Empresa de Pequeno Porte: Um Estudo de Caso na Cidade de Macapá. 2015. 18p. (Graduação). Estácio de Sá, Macapá AP.

SILVA, É. B. Conciliação Judicial. Brasília DF: Gazeta Juridica, 2013. 432p.

SILVA, P. G. A Conciliação como Instrumento de Solução de Conflitos. 2012. (Graduação). Centro Universitário Eurípides de Marília - UNIVEM, Marília SP.

TJAP. Sistema TUCUJURIS - Processo Judicial Eletrônico. Macapá AP, 2016. Disponível em: < http://www.tjap.jus.br/portal/sobre.html >. Acesso em: 22/02/2016.

TOLENTINO, A. V. A competência do Juizado Especial Cível. 2011. Disponível em: $<\quad$ http://www.direitonet.com.br/artigos/exibir/6387/A-competencia-do-JuizadoEspecial-Civel >. Acesso em: 30/05/2016.

Enviado: Mayo de 2021.

Aprobado: Mayo de 2021. 